\title{
EGOROFF'S THEOREM AND THE DISTRIBUTION OF STANDARD POINTS IN A NONSTANDARD MODEL ${ }^{1}$
}

\author{
C. WARD HENSON AND FRANK WATTENBERG
}

\begin{abstract}
We study the relationship between the Loeb measure ${ }^{0}\left({ }^{*} \mu\right)$ of a set $E$ and the $\mu$-measure of the set $S(E)=\left\{\left.x\right|^{*} x \in E\right\}$ of standard points in $E$. If $E$ is in the $\sigma$-algebra generated by the standard sets, then ${ }^{0}\left({ }^{*} \mu\right)(E)=\mu(S(E))$. This is used to give a short nonstandard proof of Egoroff's Theorem. If $E$ is an internal, * measurable set, then in general there is no relationship between the measures of $S(E)$ and $E$. However, if ${ }^{*} X$ is an ultrapower constructed using a minimal ultrafilter on $\omega$, then ${ }^{*} \mu(E) \approx 0$ implies that $S(E)$ is a $\mu$-null set. If, in addition, $\mu$ is a Borel measure on a compact metric space and $E$ is a Loeb measurable set, then

$$
\mu(S(E))<{ }^{0}\left({ }^{*} \mu\right)(E)<\bar{\mu}(S(E))
$$
\end{abstract}

where $\underline{\mu}$ and $\bar{\mu}$ are the inner and outer measures for $\mu$.

The work in this paper was originally stimulated by the search for an illuminating nonstandard proof of Egoroff's Theorem. Despite the importance of such a proof it has been surprisingly elusive (see, for example, [8] or [11]). §I of this paper presents a short, natural proof of Egoroff's Theorem using a result from §II on the distribution of standard points in a nonstandard model. The work in $\S I I$ is of independent interest.

Throughout this paper $(X, \mathscr{F}, \mu)$ will denote a (standard) positive measure space with $\mu(X)$ finite; $\mathfrak{T}$ will denote a standard higher order model of $X$ along with the real numbers, $R$; and $* \mathfrak{T}$ will denote a proper nonstandard extension of $\mathfrak{T}$. We will always assume $* \mathfrak{T}$ is $\boldsymbol{\aleph}_{1}$-saturated, but any further assumptions will be explicitly stated. If $P$ is an entity in $\Re,{ }^{*} P$ will denote the corresponding entity in * $\mathfrak{T}$. Thus, in particular, ${ }^{*} \mu:{ }^{*} \mathscr{F} \rightarrow{ }^{*}[0, \infty)$ denotes the extension in $* \mathfrak{T}$ of $\mu$ to the ${ }^{*}$ measurable sets. We use the usual notation $\operatorname{St}(x)$ for the standard part of a finite nonstandard real and $x \approx y$ for $x$ infinitely close to $y$.

I. Egoroff's Theorem. Suppose $f_{1}, f_{2}, \ldots$ is a standard sequence of measurable functions $X \rightarrow R$ and $f: X \rightarrow R$ is also a measurable function. Egoroff's Theorem [3] states

I.1 EgOROFF's THEOREM. If $f_{n} \rightarrow f$ pointwise almost everywhere then for every $\varepsilon>0$ there is a set $A \in \mathscr{F}$ such that $\mu(A)<\varepsilon$ and $f_{n} \rightarrow f$ uniformly on $X \backslash A$.

Received by the editors July 30, 1979 and, in revised form, February 5, 1980; presented to the Society, January 4, 1980 at the Special Session on Nonstandard Analysis, San Antonio, Texas.

1980 Mathematics Subject Classification. Primary 03H05.

${ }^{1}$ This work was partially supported by grants from the National Science Foundation. 
If $\left\{f_{n}\right\}$ satisfies the conclusion of Egoroff's Theorem we will say $\left\{f_{n}\right\}$ converges to $f$ nearly uniformly. Note that Egoroff's Theorem is false without the assumption that $\mu(X)$ is finite.

The following characterization of nearly uniform convergence is essentially due to Robinson [8].

I.2 Definition. Suppose $f, f_{1}, f_{2}, \ldots$ are standard measurable functions $X \rightarrow R$, and $x \in{ }^{*} X$. Then $x$ is said to be a point of intrinsic nonuniformity if there is an infinite integer $\nu$ such that $f_{\nu}(x) \approx \approx^{*} f(x)$. Let $E$ denote the set of points of intrinsic nonuniformity. (Note: $E$ is usually external.)

I.3 Definition. Suppose $A$ is a (possibly external) subset of ${ }^{*} X . A$ is said to have $S$-measure zero if for every standard $\varepsilon>0$ there is a standard set $B \in \mathcal{F}$ such that $A \subseteq \subseteq^{*} B$ and $\mu(B)<\varepsilon$.

I.4 Proposition. Using the notation of Definition I.2, the following are equivalent.

(i) $\left\{f_{n}\right\}$ converges to $f$ nearly uniformly.

(ii) $E$ has $S$-measure zero.

Proof. The proof is completely straightforward using the well-known fact that $f_{n} \rightarrow f$ uniformly on a set $S$ if and only if for every $p \in{ }^{*} S$ and every infinite $\nu$, $f_{v}(p) \approx^{*} f(p)[8$, Theorem 4.6.1].

We need one more definition before proving Egoroff's Theorem.

I.5 Definition. Suppose $A$ is a (possibly external) subset of ${ }^{*} X$. Let $S(A)$ denote the set of all standard points in $A$. That is, $S(A)=\left\{\left.x \in X\right|^{*} x \in A\right\}$. Note $S(A)$ is just the standard part of $A$ with respect to the discrete topology on $X$.

I.6 Proof of Egoroff's Theorem. Suppose $f_{n} \rightarrow f$ pointwise almost everywhere. Hence there is a set $A \in \mathcal{F}$ such that $\mu(A)=0$ and $f_{n} \rightarrow f$ pointwise on $X \backslash A$. Let $E$ denote the set of points of intrinsic nonuniformity. Then $S(E) \subseteq A$. Thus $S(E)$ has measure zero and by II.3 $E$ has $S$-measure zero, completing the proof by I.4.

II. The distribution of standard points in ${ }^{*} X$. The purpose of this section is to study the relationship between the measure (in a sense to be defined below) of a set $E \subseteq \subseteq^{*} X$ and the standard measure of $S(E)$. Intuitively, the standard points are evenly distributed in ${ }^{*} X$ and one might, therefore, expect the measures of $E$ and $S(E)$ to be infinitely close for a reasonable class of sets $E$.

II.1 Definition. Let $\mathcal{Q}$ be the (external) algebra, $\mathcal{Q}=\left\{{ }^{*} A \mid A \in \mathcal{F}\right\}$, and let $\mathcal{S}$ be the (external) $\sigma$-algebra generated by $\mathcal{Q}$. Using the Loeb-Caratheodory extension process there is an (external) real-valued $\sigma$-additive measure ${ }^{0}\left({ }^{*} \mu\right): \mathcal{S} \rightarrow[0, \infty)[5]$, see also $[8, \S 5.1]$, called $S$-measure. Notice ${ }^{0}\left({ }^{*} \mu\right)(A)=0$ if and only if $A$ has $S$-measure zero in the sense of I.3.

II.2 Theorem. Suppose $E \in \mathcal{S}$. Then $S(E) \in \mathscr{F}$ and ${ }^{0}\left({ }^{*} \mu\right)(E)=\mu(S(E))$.

Proof. First, let $\mathcal{T}_{1}=\{E \in \mathcal{S} \mid S(E) \in \mathcal{F}\}$. $\mathcal{T}_{1}$ is a $\sigma$-algebra since $S\left({ }^{*} X \backslash A\right)=$ $X \backslash S(A), S\left(A_{1} \cap A_{2}\right)=S\left(A_{1}\right) \cap S\left(A_{2}\right)$ and $S\left(\cup_{n=1}^{\infty} A_{n}\right)=\cup_{n=1}^{\infty} S\left(A_{n}\right)$. Hence $\mathcal{S}=\mathcal{T}_{1}$. Now we have two finite measures defined on $\mathcal{\delta}, \mu_{1}(E)={ }^{0}\left({ }^{*} \mu\right)(E)$ and $\mu_{2}(E)=\mu(S(E))$. By the uniqueness part of the Caratheodory Extension Theorem, we have $\mu_{1}=\mu_{2}$ completing the proof. Notice the importance here that $\mu(X)$ is finite. 
II.3 ExAMPLE. Let $E$ be as in I.6, the set of points of intrinsic nonuniformity for $f$ and $\left(f_{n}\right)$ where $f_{n} \rightarrow f$ almost everywhere. Then $E \in \mathcal{S}$ and ${ }^{0}\left({ }^{*} \mu\right)(E)=\mu(S(E))=$ 0 .

Proof. Let $A_{n, k}=\left\{x \in X|\exists r \geqslant k| f_{r}(x)-{ }^{*} f(x) \mid>1 / n\right\}$. Claim:

$$
E=\bigcup_{n=1}^{\infty} \bigcap_{k=1}^{\infty} * A_{n, k}
$$

Proof of Claim. If $x \in E$ then there is an infinite $\rho$ such that $f_{\rho}(x) \approx^{*} f(x)$. Therefore, there is a finite $n$ such that $\left|f_{\rho}(x)-{ }^{*} f(x)\right|>1 / n$. Thus $x \in A_{n, \rho}$ $\subseteq^{*} A_{n, k}$ for every finite $k . \quad \therefore x \in \cap{ }_{k=1}^{\infty}{ }^{*} A_{n, k}$. Conversely, suppose

$$
x \in \bigcup_{n=1}^{\infty} \bigcap_{k=1}^{\infty} * A_{n, k} \text {. }
$$

Therefore there is an $n$ such that $x \in \cap^{\infty}{ }_{k=1}^{*} A_{n, k}$. Let $T=\left\{k|| f_{k}(x)-{ }^{*} f(x) \mid>\right.$ $1 / n\} . T$ is internal and contains arbitrarily large finite positive integers $k$. Therefore $T$ contains some infinite positive integer $\nu$ and $\left|f_{\nu}(x)-{ }^{*} f(x)\right|>1 / n$. So $x \in E$.

One of the difficulties in applying the techiques of Nonstantard Analysis to standard problems is converting a nonstandard object into a standard one. In particular, if $F:{ }^{*} X \rightarrow \mathbf{R}$ is Loeb measurable and we define $f: X \rightarrow \mathbf{R}$ by $f(x)=$ $F(x)$ then we have very little control over $f$. In fact, $f$ need not be measurable and * $f$ need have little relationship to $F$. One consequence of Theorem II.2 is that the situation is much better if $F$ is $\mathcal{S}$-measurable. More precisely, we have

II.4 Theorem. Suppose $F:{ }^{*} X \rightarrow \mathbf{R}$ is $\mathcal{S}$-measurable and $f: X \rightarrow \mathbf{R}$ is defined by $f(x)=F(x)$. Then

(1) $f$ is $\mathscr{F}$-measurable,

(2) $\left\{\left.x \in{ }^{*} X\right|^{*} f(x) \approx F(x)\right\}$ has $S$-measure zero.

Proof. (1) Let $t \in \mathbf{R}$ and $A=\{x \mid f(x)>t\}$. Notice $A=S(E)$ where $E=$ $\{x \mid F(x)>t\} \in \mathcal{S}$ by assumption. Hence, by Theorem II.2 $A \in \mathcal{F}$ and $f$ is $\mathscr{F}$-measurable.

(2) Let ${ }^{0}\left({ }^{*} f\right):{ }^{*} X \rightarrow \mathbf{R} \cup\{\infty\}$ be given by

$$
{ }^{0}(f)(x)= \begin{cases}\left.\operatorname{St}^{*} f(x)\right) & \text { if } * f(x) \text { is finite, } \\ \infty & \text { if } * f(x) \text { is infinite. }\end{cases}
$$

A straightforward argument shows ${ }^{0}\left({ }^{*} f\right)$ is $\mathcal{S}$-measurable. Let $E=\left\{\left.x\right|^{0}\left({ }^{*} f\right)(x) \neq\right.$ $F(x)\}$. Then $E \in \mathcal{S}$ and $S(E)=\varnothing$. So, $E$ has $S$-measure zero by Theorem II.2. But $E=\left\{\left.x\right|^{*} f(x) \approx F(x)\right\}$ completing the proof.

The obvious question to ask is whether Theorem II.2 can be extended to a larger class of sets. A natural such question is whether for internal *measurable sets $E$, ${ }^{*} \mu(E) \approx \mu(S(E))$. Unfortunately, the possible results in this direction are sharply circumscribed by the following examples.

II.5 Example. Suppose $X=[0,1]$ and ${ }^{*} X$ is an enlargement of $X$. Then for every $B \subseteq[0,1]$ and $t \in{ }^{*}[0,1]$ there is an internal, ${ }^{*}$ Borel set $E$ such that $S(E)=B$ and ${ }^{*} \mu(E)=t$. 
Proof. A straightforward enlargement argument produces *finite sets $F_{1}, F_{2}$ such that $S\left(F_{1}\right)=B, S\left(F_{2}\right)=[0,1] \backslash B$ and $F_{1} \cap F_{2}=\varnothing$. Let

$$
E=\left(*[0, t] \cup F_{1}\right) \backslash F_{2} \text {. }
$$

II.6 Example. Suppose $X=\{0,1\}^{(\infty)}$ (i.e. an element $x \in X$ is a sequence $x=\left(x_{1}, x_{2}, \ldots\right)$ of 0 's and 1's). Let $X$ have the obvious probability measure. Let ${ }^{*} X$ be any proper nonstandard extension of $X$ and let $\nu$ be any infinite positive integer. Let $E=\left\{x \in{ }^{*} X \mid x_{\nu}=1\right\}$. It is well known and easy to prove using the Kolmogorov Zero-One Law that $S(E)$ has inner measure zero and outer measure one. But ${ }^{*} \mu(E)=1 / 2$.

II.7 EXAMPLE. We construct a nonstandard model $* \mathfrak{R}=\Re^{J} / D$, where $J$ is countable, such that there is an internal, *open set $V \subseteq{ }^{*}[0,1]$ with $S(V)=\varnothing$ but ${ }^{*} \mu(V) \approx 1$ ( $\mu$ is Lebesgue measure). Let $X=[0,1]$ and let $J$ be the set of all finite unions of disjoint open intervals with rational endpoints. Thus a typical element $U$ of $J$ is a set $\cup_{n=1}^{k}\left(a_{n}, b_{n}\right)$ with $a_{1}<b_{1}<a_{2}<\cdots<b_{n}$ all rational. Notice $J$ is countable. If $x_{1}, x_{2}, \ldots, x_{k} \in[0,1]$ let $\mathscr{Q}\left(x_{1}, x_{2}, \ldots, x_{k}\right)=\{U \in$ $\left.J \mid x_{1}, x_{2}, \ldots, x_{k} \notin U, \mu(U)>1-1 / k\right\}$. Clearly the sets $\mathcal{U}\left(x_{1}, x_{2}, \ldots, x_{k}\right)$ are nonempty and have the finite intersection property. Let $D$ be any ultrafilter containing all the sets $\mathscr{U}\left(x_{1}, x_{2}, \ldots, x_{k}\right)$. Let $* \mathscr{R}=\mathfrak{N}^{J} / D$ and let $V \subseteq \subseteq^{*} X$ be the *open set represented by the function $F: J \rightarrow P(X)$ given by $F(U)=U$. It is immediate from the construction of $V$ that ${ }^{*} \mu(V) \approx 1$ and $S(V)=\varnothing$.

Thus, in general, there is no relationship between ${ }^{*} \mu(E)$ and $\mu(S(E))$. However, if ${ }^{*} X$ is a minimal nonstandard model (defined below) we do have some positive results.

II.8 Definition. Suppose $J=\{1,2,3, \ldots\}$ and $D$ is an ultrafilter on $J . D$ is said to be minimal, see [1], [9], [10], provided whenever $f: J \rightarrow J$ there is a set $A \in D$ such that either $f$ is constant on $A$ or $f$ is one-to-one on $A$. If either the Continuum Hypothesis or Martin's Axiom holds there are many minimal ultrafilters on $J$ [1], [9], [10]. If $D$ is a minimal ultrafilter on $J$, the nonstandard model $\mathfrak{N}^{J} / D$ is said to be minimal. We use below the fact that minimal ultrafilters are Ramsey [2] and therefore satisfy the strong Ramsey theorem proved by Mathias [6], [7].

II.9 Proposition. Suppose * $\mathfrak{N}$ is a minimal nonstandard model and $E \subseteq{ }^{*} X$ is an internal ${ }^{*}$ measurable set such that ${ }^{*} \mu(E) \approx 0$. Then $S(E)$ is a $\mu$-null set.

Proof. Let $e={ }^{*} \mu(E)$, let $\left(E_{1}, E_{2}, \ldots\right)$ represent $E$ and let $e_{n}=\mu\left(E_{n}\right)$. Hence, $e$ is represented by $\left(e_{1}, e_{2}, \ldots\right)$. Define $f: J \rightarrow J$ by $f(n)=$ largest $k$ such that $e_{n} \leqslant 1 / 2^{k}$. Since $D$ is minimal there is a set $A \in D$ such that $f$ is constant on $A$ or $f$ is one-to-one on $A$. Since $e \approx 0$ the first alternative is impossible. Hence $f$ is one-to-one on $A$. Therefore

$$
\sum_{n \in A} e_{n} \leqslant \sum_{k=1}^{\infty} 1 / 2^{k}=1 .
$$

Now, suppose $\varepsilon>0$ is standard. Then there is a set $B \in D$ such that

$$
\sum_{n \in B} e_{n}<\varepsilon
$$


But $x \in S(E)$ implies $x \in \cup_{r \in B} E_{n}$. Thus $S(E) \subseteq \bigcup_{n \in B} E_{n}$ but $\mu\left(\cup_{n \in B} E_{n}\right)<$ $\sum_{n \in B} e_{n} \leqslant \varepsilon$. This completes the proof.

If $* \mathfrak{T}$ is a minimal nonstandard model, $\mu$ is a Borel measure on a compact metric space and $E$ is Loeb measurable then considerably more can be said about the relationship between the measures of $E$ and $S(E)$. The first step is the following lemma.

II.10 Lemma. Suppose * $\mathfrak{T}$ is minimal nonstandard model, $\mu$ is a Borel measure on a compact metric space $K$ and $E$ is a Loeb measurable set such that $S(E)=\varnothing$. Then ${ }^{0}\left({ }^{*} \mu\right)(E)=0$.

Proof. Suppose $E$ is Loeb measurable, $S(E)=\varnothing$ and ${ }^{0}\left({ }^{*} \mu\right)(E)>0$. Then there is a standard $\varepsilon>0$ and an internal, ${ }^{*}$ measurable set $F \subseteq E$ such that ${ }^{*} \mu(F)>\varepsilon$. Let $\left(F_{n}\right)$ be a sequence of Borel subsets of $K$ which determines $F$ as an element of $* \mathfrak{T}$. We then have that the set $Y=\left\{n \mid \mu\left(F_{n}\right)>\varepsilon\right\}$ is an element of the minimal ultrafilter $D$ which is used to construct $* \mathfrak{N}$. The Ramsey Theorem for $D$ due to Mathias [6] will be used to show that there exists $Z \in D$ with $Z \subseteq Y$ and

$$
\cap\left\{F_{n} \mid n \in Z\right\} \neq \varnothing \text {. }
$$

Since this intersection is contained in $S(F)$, this contradicts our assumption that $S(F)=\varnothing$.

Given an infinite set $W \subseteq \mathbf{N}$, set $[W]^{\omega}=\{V \mid V$ is an infinite subset of $W\}$. On $[N]^{\omega}$ put the usual topology: the basic open neighborhoods of $W \in[N]^{\omega}$ are the sets

$$
\left\{V \in[N]^{\omega} \forall k<n(k \in V \leftrightarrow k \in W)\right\}
$$

for $n=1,2, \ldots$ The Ramsey Theorem of Mathias implies that if $R \subseteq[N]^{\omega}$ is analytic relative to this topology, there exists some $W \in D$ such that either $[W]^{\omega} \subseteq \Re$ or $[W]^{\omega} \cap \Re=\varnothing$. (By [2] a minimal ultrafilter is Ramsey; for a proof of Mathias' result that Ramsey ultrafilters have this much stronger property see [7].)

For our purposes we use the family

$$
\Re=\left\{W \in[N]^{\omega} \mid \cap\left\{F_{n} \mid n \in W\right\} \neq \varnothing\right\} .
$$

First we show that $R$ is analytic. Consider the set $\delta \subseteq[N]^{\omega} \times K$ defined by

$$
\mathcal{S}=\left\{(\boldsymbol{W}, x) \mid x \in \cap\left\{F_{n} \mid n \in W\right\}\right\} \text {. }
$$

Since each $F_{n}$ is a Borel subset of $K, \mathcal{S}$ is a Borel set in the product space $[N]^{\omega} \times K$. Also $R$ is the image of $\delta$ under the coordinate projection from $[N]^{\omega} \times K$ onto $[N]^{\omega}$. Since $K$ is a compact metric space, it follows that $R$ is analytic in $[N]^{\omega}[4$, Chapter XIII].

Now apply Mathias' theorem, obtaining a set $W \in D$ such that $[W]^{\omega} \subseteq \Re$ or $[W]^{\omega} \cap \Re=\varnothing$. In the first case we have $Z=Y \cap W \in D$ and $\cap\left\{F_{n} \mid n \in Z\right\}$ $\neq \varnothing$ as desired. Thus it suffices to prove the second case is impossible. For any $W \in D$ the set $Z=Y \cap W$ is infinite and $\mu\left(F_{n}\right)>\varepsilon>0$ holds for every $n \in Z$. Since $\mu$ is a finite measure it follows that

$$
\mu\left(\bigcap_{n \in N} \bigcup\left\{F_{k} \mid k \in Z \text { and } k>n\right\}\right)>\varepsilon .
$$


Thus we may choose $x$ and an infinite subset $V$ of $Z$ with $x \in F_{k}$ for all $k \in V$. Therefore $V \in[W]^{\omega} \cap \mathcal{R}$, which completes the proof.

II.11 REMARK. In case $\mu$ is a regular Borel measure on $K$, a relatively simple case of Mathias' theorem can be used in the proof of II.10. In that case we may assume that the sets $F_{n}$ are closed (replacing $\varepsilon$ by $\varepsilon / 2$ and each $F_{n}$ by a closed subset). Then the family $R$ is actually a closed subset of $[N]^{\omega}$ as can be seen by a direct proof.

II.12 Theorem. Suppose * $\Re$ is a minimal nonstandard model and $\mu$ is a Borel measure on a compact metric space $K$. If $E \subseteq{ }^{*} K$ is Loeb measurable then

$$
\underline{\mu}(S(E)) \leqslant^{0}\left({ }^{*} \mu(E)\right) \leqslant \bar{\mu}(S(E))
$$

where $\mu, \bar{\mu}$ are the inner and outer measures for $\mu$.

Proof. It suffices to prove $\mu(S(E)) \leqslant^{0}\left(^{*} \mu(E)\right)$ since the other inequality follows from this one applied to ${ }^{*} K \backslash \bar{E}$.

Let $B$ be a standard measurable set such that $B \subseteq S(E)$ and $\mu(B)=\mu(S(E))$. Let $A={ }^{*} B \backslash E$. Notice $S(A)=\varnothing$ and $A$ is Loeb measurable so by Lemma II.10 ${ }^{0}\left({ }^{*} \mu\right)(A)=0$. Hence since ${ }^{0}\left({ }^{*} \mu\right)(A)+{ }^{0}\left({ }^{*} \mu\right)(E) \geqslant{ }^{0}\left({ }^{*} \mu\right)(B)=\mu(S(E))$ we have ${ }^{0}\left({ }^{*} \mu\right)(E) \geqslant \mu(S(E))$ completing the proof.

Example $\bar{I} .6$ shows that even if $E$ is internal and *measurable, $S(E)$ need not be $\mu$-measurable. The following Corollary gives a necessary and sufficient condition for $S(E)$ to be $\mu$-measurable when $E$ is an internal, *Borel set in a minimal nonstandard model $* \Re$.

II.13 Corollary. Let $* \mathfrak{T}, \mu$ and $K$ be as in II.10. For each internal ${ }^{*}$ Borel set $E \subseteq \subseteq^{*} K, S(E)$ is measurable with respect to the completion of $\mu$ if and only if there is $a$ standard Borel set $B$ such that $E$ and ${ }^{*} B$ differ by a set of infinitesimal ${ }^{*} \mu$ measure.

Proof. If such a set $B$ exists, then $S(E)$ equals $B$ up to a $\mu$-null set, by II.9.

For the converse, suppose $S(E)$ is measurable with respect to the completion of $\mu$ and let $B \subseteq S(E)$ be a Borel set such that $S(E) \backslash B$ is a $\mu$ null set. Then $S\left({ }^{*} B \backslash E\right)$ is empty and $S\left(E \backslash{ }^{*} B\right)$ is a $\mu$-null set, so that the symmetric difference of ${ }^{*} B$ and $E$ has infinitesimal ${ }^{*} \mu$ measure, by II. 12 .

II.14 Remark. Some restriction on the measure space of $\mu$ is necessary in order that II.10 should be true. For example, take Borel subsets $E_{n}$ of $\{0,1\}^{(\infty)}$ as in Example II.6, so that the internal set $E$ determined by $\left(E_{n}\right)$ has internal measure $1 / 2$, yet $S(E)$ has inner measure 0 and outer measure 1 . Then consider the measure space $\Omega=\{0,1\}^{(\infty)} \backslash S(E)$ with the restricted measure $\mu$. Let $E_{n}^{\prime}=E_{n} \backslash S(E)$, so $\left(E_{n}^{\prime}\right)$ are measurable subsets of $\Omega$. If $E^{\prime}$ is the internal *measurable subset of ${ }^{*} \Omega$ determined by the sequence $\left(E_{n}^{\prime}\right)$, then $S\left(E^{\prime}\right)=\varnothing$ yet ${ }^{*} \mu\left(E^{\prime}\right)=1 / 2$.

II.15 RemarK. Lemma II.10 which is the key step in the proof of Theorem II.12 has a very nice standard interpretation as follows. Suppose $D$ is a minimal ultrafilter on the set $\{1,2,3, \ldots\}$ and $E_{1}, E_{2}, \ldots$ is a sequence of Borel subsets of a compact metric space $K$ with $\mu$ a finite Borel measure on $K$. If inf $\mu\left(E_{n}\right)>0$ then there is a point $x \in K$ such that $\left\{n \mid x \in E_{n}\right\} \in D$. Notice this is a strengthening of the usual result that there is a point $x \in K$ which is in infinitely many $E_{n}$ 's. 


\section{REFERENCES}

1. A. Blass, The Rudin-Keisler ordering of P-points, Trans. Amer. Math. Soc. 179 (1973), 145-166.

2. D. Booth, Ultrafilters on a countable set, Ann. Math. Logic 2 (1970), 1-24.

3. P. R. Halmos, Measure theory, Van Nostrand, Princeton, N.J., 1950.

4. K. Kuratowski and A. Mostowski, Set theory with an introducton to descriptive set theory, North-Holland, Amsterdam, 1976.

5. P. Loeb, Conversion from nonstandard to standard measure spaces and applications to probability theory, Trans. Amer. Math. Soc. 211 (1975), 113-122.

6. A. R. D. Mathias, Solution of problems of Choquet and Puritz, Conference in Mathematical Logic '70, Lecture Notes in Math., vol. 255, Springer-Verlag, Berlin and New York, 1972, pp. 204-210.

7. K. R. Milliken, Completely separable families and Ramsey's theorem, J. Combinatorial Theory 19 (1975), 318-334.

8. A. Robinson, Non-standard analysis, North-Holland, Amsterdam, 1974.

9. M. Rudin, Types of ultrafilters, Topology Seminar, Wisconsin, R. H. Bing and R. J. Bean (eds.), Princeton Univ. Press, Princeton, N.J., 1966.

10. W. Rudin, Homogeneity problems in the theory of $\check{C}$ ech compactifications, Duke Math. J. 23 (1956), 409-420.

11. K. D. Stroyan and W. A. J. Luxemburg, Introduction to the theory of infinitesimals, Academic Press, New York, 1976.

Department of Mathematics, University of Illinois at Urbana-Champaign, Urbana, Illinois 61801

Department of Mathematics, University of Massachusetts, Amherst, Massachusetts 01003 